\title{
Sobre a Dificuldade de Reconhecimento de Grafos $B_{1}$-EPG-Helly
}

\author{
Claudson Bornstein ${ }^{1}$, Tanilson Santos ${ }^{1,2}$, Uéverton Souza ${ }^{3}$, Jayme Szwarcfiter $^{1,4}$ \\ ${ }^{1}$ Programa de Engenharia de Sistemas e Computação - UFRJ (Coppe/PESC) \\ Caixa Postal 68511 - 21941-972 - Rio de Janeiro - RJ - Brasil \\ ${ }^{2}$ Curso de Ciência da Computação - Universidade Federal do Tocantins - UFT \\ Palmas-TO, Brasil \\ ${ }^{3}$ Instituto de Computação - Universidade Federal do Fluminense - UFF \\ Niterói, Brasil \\ ${ }^{4}$ Departamento de Informática e Ciência da Computação - IME \\ Universidade do Estado do Rio de Janeiro - UERJ - Rio de Janeiro, RJ - Brasil \\ \{cbornstein, uevertonssouza\}@gmail.com, \{jayme, tanilson\}@cos.ufrj.br
}

\begin{abstract}
Golumbic, Lipshteyn e Stern have defined in 2009 the EPG graphs, a intersection graph class based on edge intersection of paths on a grid. An EPG graph $G$ is a graph that admits a representation scheme where its vertices are represented by paths on a grid $Q$, such that two vertices of $G$ are adjacent if and only if their corresponding paths in $Q$ have a common edge. If the paths in $Q$ have at most $k$ changes of direction (bends), we say that this is a $B_{k^{-}}$EPG representation. A collection $C$ of sets satisfies the Helly property when every sub-collection of $C$ that is pairwise intersecting has at least one common element. In this paper we show that the problem of recognizing $B_{1}-E P G$ graphs that satisfy the Helly property is NP-hard.
\end{abstract}

Resumo. Golumbic, Lipshteyn e Stern definiram em 2009 os grafos EPG, uma classe de grafos de intersecção baseada na intersecção de arestas de caminhos em uma grade. Um grafo EPG G é um grafo que admite um esquema de representação onde seus vértices são representados por caminhos de uma grade $Q$, de forma que dois vértices em $G$ são adjacentes se e somente se os caminhos correspondentes em $Q$ compartilham arestas. Se tais caminhos em $Q$ tem no máximo $k$ mudanças de direção (dobras), então a representação é dita $B_{k^{-}}$ EPG. Uma coleção $C$ de conjuntos satisfaz a propriedade Helly quando toda subcoleção de C que é mutuamente intersectante possui pelo menos um elemento comum. Neste trabalho mostramos que o reconhecimento de grafos que admitem representação $B_{1}$-EPG satisfazendo a propriedade Helly é NP-difícil.

\section{Introdução}

Um grafo EPG $G$ é um grafo que admite um esquema de representação onde seus vértices são representados por caminhos de uma grade $Q$, de tal forma que dois vértices em $G$ são adjacentes se e somente os caminhos correspondentes em $Q$ possuem arestas em comum. O estudo de grafos EPG tem motivação prática relacionada ao problema de layout de 
circuitos digitais, que podem ser representados como caminhos em uma grade ortogonal retangular.

Todo grafo $G$ possui uma representação EPG, onde cada vértice $v$ é representado como um caminho $p(v)$ e dois vértices $u, v$ são adjacentes se e somente se $p(u) \cap p(v) \neq \emptyset$, ver [Golumbic et al. 2009]. Um grafo possui uma representação $B_{k}$-EPG se existe uma representação para o grafo na qual cada caminho tem no máximo $k$ dobras.

A classe de grafos EPG tem sido estudada por diversos pesquisadores, atualmente, como [Ries 2009, Alcón et al. 2016, Cohen et al. 2014], entre outros. Essas pesquisas abordam com frequência caracterizações com relação a quantidade de dobras na representação dos grafos. Com relação à complexidade de reconhecimento, somente 3 classes de grafos EPG estão determinadas: $B_{0}$-EPG [Golumbic et al. 2009], $B_{1}$-EPG [Heldt et al. 2014] e mais recentemente $B_{2}$-EPG [Martin Pergel 2017], sendo somente $B_{0}$-EPG reconhecido em tempo polinomial, pois corresponde à classe de grafos de intervalo, ver [Booth and Lueker 1976]. A título de exemplo, a Figura 1(a) esboça o grafo $C_{3}$, na Figura 1(b) é exibida uma representação EPG onde os caminhos não possuem dobra, na Figura 1(c) uma representação com 1 dobra e na Figura 1(d) uma representação onde todo caminho possui no máximo 2 dobras.

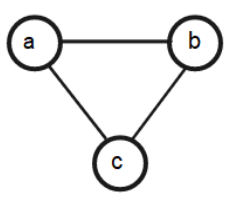

(a) Grafo $C_{3}$

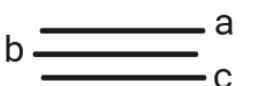

(b) Representação $B_{0}-\operatorname{EPG}\left(C_{3}\right)$

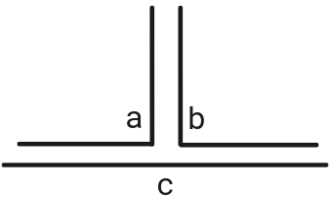

(c) Representação $B_{1}-\operatorname{EPG}\left(C_{3}\right)$

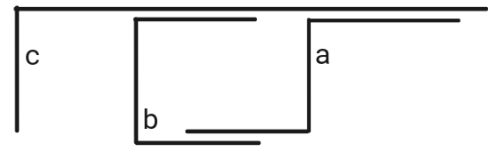

(d) Representação $B_{2}-\operatorname{EPG}\left(C_{3}\right)$

Figura 1. Grafo $C_{3}$ e algumas de suas representações sem dobra, com 1 dobra e com 2 dobras

Uma coleção $C$ de conjuntos satisfaz a propriedade Helly quando toda subcoleção de $C$ que é mutuamente intersectante possui pelo menos um elemento comum. A propriedade Helly tem esse nome em homenagem ao grande matemático austríaco Eduard Helly, que em 1923 propôs seu famoso teorema a respeito do relacionamento de conjuntos intersectantes.

Este trabalho trata de um estudo sobre grafos de intersecção de arestas em grade que possuem a propriedade Helly em sua representação. Nossa contribuição é apresentar uma prova da NP-dificuldade relacionada ao reconhecimento de grafos $B_{1}$-EPG-Helly.

\section{As classes $B_{1}$-EPG e $B_{1}$-EPG-Helly}

Embora as classes $B_{1}$-EPG e $B_{1}$-EPG-Helly não coincidam, o mesmo não ocorre entre as classes $B_{0}$-EPG e $B_{0}$-EPG-Helly que coincidem, como observado no Lema 1.

Lema 1. Toda representação $B_{0}-E P G$ satisfaz a propriedade Helly.

Corolário 2. A classe de grafos $B_{0}-E P G$ coincide com a classe $B_{0}-E P G$-Helly.

Definição 3. [Golumbic et al. 2009] Dada uma coleção de caminhos $P=\left\{p_{1}, \ldots, p_{4}\right\}$ de uma grade $Q$ em uma representação $B_{1}-E P G$ do grafo $G$, considere um subgrafo 4-estrela de $Q$ cujo centro está posicionado sobre o ponto $b$ e as arestas $\left(a_{1}, b\right),\left(a_{2}, b\right),\left(a_{3}, b\right),\left(a_{4}, b\right)$, tomadas em sentido horário. Define-se: 
- Uma torta verdadeira é uma 4-estrela onde cada segmento $\left(a_{i}, b\right) \cup\left(a_{i+1}, b\right)$, para $1 \leq i \leq 4$, é um membro diferente de $P$, onde adicionalmente é assumido ser módulo 4. Todos os 4 caminhos de G fazem dobra em $b$.

- Uma torta falsa é uma 4-estrela onde cada segmento $\left(a_{1}, b\right) \cup\left(a_{2}, b\right) ;\left(a_{2}, b\right) \cup$ $\left(a_{4}, b\right) ;\left(a_{4}, b\right) \cup\left(a_{3}, b\right) ;\left(a_{3}, b\right) \cup\left(a_{1}, b\right)$, para $1 \leq i \leq 4$, é um membro diferente de P. Na torta falsa, somente 2 caminhos, não adjacentes entre si, fazem dobra em $b$.

- Considerando um retângulo de qualquer tamanho com 4 cantos $\left(x_{1}, y_{1}\right) ;\left(x_{2}, y_{1}\right) ;\left(x_{2}, y_{2}\right) ;\left(x_{1}, y_{2}\right)$. Um quadro ou moldura é um retângulo em que em cada canto está uma dobra diferente para cada membro de $p_{1}, \ldots, p_{4} \in P$. Os subcaminhos $p_{1} \cap p_{2}, p_{2} \cap p_{3}, p_{3} \cap p_{4}, p_{4} \cap p_{1}$, compartilham pelo menos uma aresta. Enquanto os subcaminhos $p_{2} \cap p_{4}$ e $p_{1} \cap p_{3}$ não compartilham aresta.
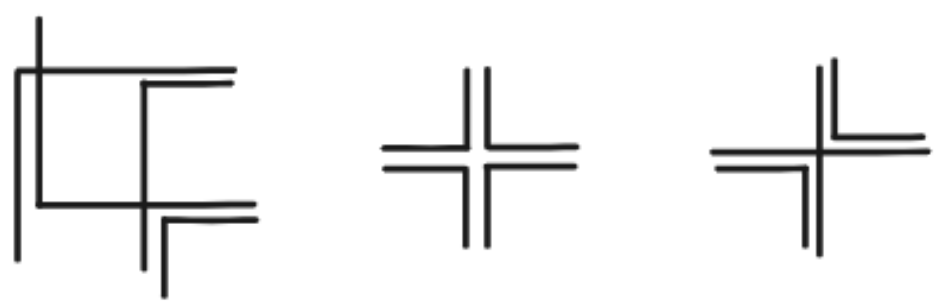

Figura 2. Representação $B_{1}$-EPG de ciclo de tamanho 4: moldura (à esquerda), torta verdadeira (centro) e torta falsa (direita), [Golumbic et al. 2009].

Lema 4. [Golumbic et al. 2009] Dada uma coleção de caminhos $P=\left\{p_{1}, \ldots, p_{4}\right\}$ de uma grade $Q$ em uma representação $B_{1}-E P G$ do grafo $G$. Todo $C_{4}$ induzido em $G$ corresponde, em qualquer representação, a uma torta verdadeira, a uma torta falsa ou a uma moldura.

Lema 5. $O$ grafo octaedro $O_{3}$ possui representação $B_{1}$-EPG $\left(O_{3}\right)$ minimal, a menos de isomorfismos.

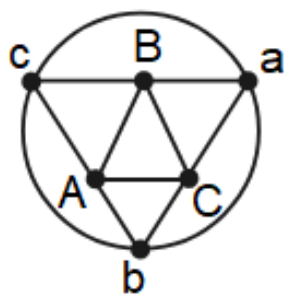

(a) Grafo octaedro $\mathrm{O}_{3}$

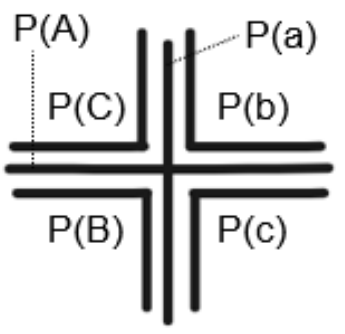

(b) Representação $B_{1}-\mathrm{EPG}\left(O_{3}\right)$

Figura 3. Grafo octaedro $O_{3}$ e sua representação $B_{1}$-EPG, [Heldt et al. 2014]

Pelo Lema 5 o grafo octaedro $O_{3}$, da Figura 3(a), é isomorfo, em qualquer representação $B_{1}$-EPG minimal, à representação da Figura 3(b). Pela Figura 3(b) podemos observar que, por exemplo, os caminhos $p(a), p(b)$ e $p(c)$ formam uma estrutura que não satisfaz a propriedade Helly. Por essa observação e da informação mostrada no Lema 5 segue o seguinte Corolário 6.

Corolário 6. A classe $B_{1}$-EPG-Helly é subclasse própria de $B_{1}-E P G$. 


\section{O Reconhecimento de $\boldsymbol{B}_{\mathbf{1}}$-EPG-Helly é NP-difícil}

A prova de NP-dificuldade de reconhecimento de $B_{1}$-EPG-Helly envolve uma redução ao problema de (1em3)-3SAT POSITIVO, similar à utilizada por [Heldt et al. 2014] para $B_{1}$-EPG. O problema relacionado é o seguinte:

\begin{tabular}{ll}
\hline \hline Instância: & \multicolumn{1}{c}{ RECONHECIMENTO DE GRAfo $G}$. \\
& Determinar se existe um conjunto de caminhos de dobra simples \\
& $P=\left\{p_{1}, p_{2}, \ldots, p_{n}\right\}$ em uma grade $Q$ representando $V(G)$ tal que: \\
Objetivo: & $\bullet u, v \in V(G)$ são adjacentes em $G$ se e somente se $p_{u}, p_{v}$ são intersectantes em $Q ;$ \\
& $\bullet \quad P$ satisfaz a propriedade de Helly. \\
\hline
\end{tabular}

A redução codifica uma fórmula $F$ pertencente a (1em3)-3SAT POSITIVO em um grafo dispositivo específico $G_{F}$. O dispositivo $G_{F}$ força que a construção de uma representação $B_{1}$-EPG-Helly que só existirá caso $F$ seja satisfatível. $G_{F}$ é subdividido em 3 partes: um dispositivo cláusula, para cada cláusula de $F$; um dispositivo variável, para cada variável em $F$; e um dispositivo base para construção da representação.

Uma fórmula $F$ pertencente a (1em3)-3SAT POSITIVO é satisfatível se e somente se existir uma $\mathrm{H}-B_{1}-\mathrm{EPG}\left(G_{F}\right)$, onde $G_{F}$ é um dispositivo construído apropriadamente a partir de $F$.

\section{Referências}

Alcón, L., Bonomo, F., Durán, G., Gutierrez, M., Mazzoleni, M. P., Ries, B., and Valencia-Pabon, M. (2016). On the bend number of circular-arc graphs as edge intersection graphs of paths on a grid. Discrete Applied Mathematics, 234:12-21.

Booth, K. and Lueker, G. (1976). Testing for the consecutive ones property, interval graphs, and graph planarity using pq-tree algorithms. Journal of Computer and System Sciences, 13:335-379.

Cohen, E., Golumbic, M. C., and Ries, B. (2014). Characterizations of cographs as intersection graphs of paths on a grid. Discrete Applied Mathematics, 178:46-57.

Golumbic, M., Lipshteyn, M., and Stern, M. (2009). Edge intersection graphs of single bend paths on a grid. Networks, 54:130-138.

Heldt, D., Knauer, K., and Ueckerdt, T. (2014). Edge-intersection graphs of grid paths: the bend-number. Discrete Appl. Math, 167:144-162.

Martin Pergel, P. R. (2017). On edge intersection graphs of paths with 2 bends. Discrete Applied Mathematics, 226:106-116.

Ries, B. (2009). Some properties of edge intersection graphs of single bend paths on a grid. Electronic Notes in Discrete Mathematics, 34:29-33. 